Purpose To review the outcomes of communities who have achieved designation as an international safe community over the 32-year period from 1986 to 2018

Method An audit of the ISCCC database and website was undertaken. Any inconsistencies were checked for accuracy against the original designation application.

Results Since the safe community movement began in 1998 four hundred and three International Safe Communities have been designated. Data was available regarding 361 communities. The program has a high reach, 86 million people have been served by a safe community program that has achieved International Safe Community Designation. Forty-nine million of these are served by safe community programs whose certification remains active.

In 2018 there were 39 certifications (13 designations and 26 re-designations), covering a population of 7.1 million people.

Conclusion The International Safe Community movement has accomplished remarkable growth in the 30 years since it began in Sweden in the late 1980s. The program has achieved high reach. Four hundred and three communities have been designated by the end of 2018 with a population footprint of 86 million people.

\section{F.004 EXPLORING POST-DISASTER HEALTH PRACTICE AMONG DISASTER-PRONE COMMUNITY IN SOUTHERN PART OF BANGLADESH}

Notan Chandra Dutta*, Ashim Kumar Saha, Shafkat Hossain, Aslam Parvej, AKM Fazlur Rahman, Aminur Rahman. Centre for Injury Prevention and Research Bangladesh, Mohakhali, Dhaka, Bangladesh

\subsection{6/injuryprev-2021-safety.57}

Background Aquatic disasters frequently affect Bangladesh. Most of these disasters occur in southern part of the country. A little is known about the post-disaster health seeking behaviors, management's role regarding disaster resilience in disaster prone communities. This study explored health-seeking behaviours, disaster resilience mechanism associated with drowning events in coastal region of Bangladesh.

Methodology A qualitative study was conducted in Barishal division from October-November 2016 to collect the required information. 12 IDIs and 4 FGDs were conducted with disaster experienced men and women, community leaders and community level disaster support volunteers. NVIVO-11 was used to organize the data. Content and thematic analysis was performed.

Results Respondents mentioned distance between their households and emergency shelters as well as poor road infrastructure obstructed them going to emergency shelters during adverse weather. Shelters were potentially unsafe for women with poor infrastructure and remain overcrowded following any natural disasters. Community people had less faith in government systems for their unequal post-disaster relief distribution including their bribery and nepotism. Government and NGOs' post-disaster relief was available for short-periods, even sporadically. In post-disaster health-seeking behavior related to drowning, people tried to apply their perception and knowledge they obtained from neighbors, elderly people. They followed traditional methods, advice from religious leader. People usually didn't go health centers for long distance from their locality.
Conclusion and Learning Outcomes Findings of this study could be an information base to develop multi-sectoral drowning prevention plan in synchronization with the existing disaster preparedness action and ensuring post-disaster health care services.

\section{$2 \mathrm{G}$ - Poisoning/Chemical, March 23, 2021}

\section{G.001 SHOULD I REALLY SPRAY THAT! SAFETY CONCERNS OF HERBICIDES AND PESTICIDES}

${ }^{1}$ Helen Truscott* ${ }^{2}$ Carol Wylie, ${ }^{2}$ John Pearn, ${ }^{1}$ Richard Franklin. 'James Cook University, Townsville, Australia; ${ }^{2}$ Queensland Poisons Information Centre, South Brisbane, Australia; ${ }^{3}$ University of Queensland, South Brisbane, Australia

10.1136/injuryprev-2021-safety.58

Background Herbicides and pesticides are commonly used in Queensland, with concentrates being severely toxic if ingested. Toxicity can be aggregative with the addition of surfactants (detergents).

Methods A systematic search of peer reviewed literature and retrospective review of the Queensland Poisons Information Centre (QPIC) data from 2015 to 2019 was conducted. Patient demographics, extent and type of exposure: accidental or non-accidental, route, initial symptoms, circumstances around exposure and subsequent treatment in hospital were analysed.

Results Each year, there are on average 35,000 calls to the QPIC, of which 1\% involve herbicides and 3.4\% pesticides. Common herbicides encountered in 2018 and 2019 include glyphosate (50\%), bromoxynil and paraquat, with common pesticides including pyrethrin/pyrethroids (29\%) and rodenticides $(16 \%)$. Where gender was known, there were more males $(52 \%)$ than females $(40 \%)$ with the most common age group being $0-4$ years, reflecting the unintentional nature of many exposures. On average, $30 \%$ of victims were already in a medical facility or advised to seek medical attention.

Conclusions Herbicides and pesticides, while intended for control of plants and pests, can also cause harm to humans, with many exposures resulting in medical intervention. Additional toxicity may result from products with multiple ingredients, affecting the advice given and medical treatment required.

A further review of the data is indicated, to explore potential risks of exposure to herbicides and pesticides to inform public awareness and safety strategies, especially for parents of young children

\section{G.002 SCOPING REVIEW OF ACUTE POISONING OBSERVATIONAL STUDIES TO IUNDERSTAND TOXICOEPIDEMIOLOGY IN AUSTRALIA}

1,2 Jared Brown*. 'NSW Poisons Information Centre, Westmead, Australia; ${ }^{2}$ UNSW, Sydney, Australia

10.1136/injuryprev-2021-safety.59

Background We reviewed Australian observational studies on acute poisoning, using an epidemiological framework, to characterise epidemiological data reported on the host, exposure and environment over time. To identify opportunities for 